\title{
A MULTI-WAVELENGTH STUDY OF THE HOST ENVIRONMENT OF SMBHB 4C+37.11
}

\author{
Roger W. Romani ${ }^{1}$, W. R. Forman ${ }^{2}$, Christine Jones $^{2}$, S. S. Murray ${ }^{3}$, \\ A. C. Readhead ${ }^{4}$, Greg B. TaYlor ${ }^{5}$, and R. T. Zavala ${ }^{6,7}$ \\ ${ }^{1}$ Department of Physics, Stanford University, Stanford, CA 94305, USA; rwr@astro.stanford.edu \\ ${ }^{2}$ Smithsonian Astrophysical Observatory, 60 Garden Street, Cambridge, MA 02138, USA \\ 3 Johns Hopkins University, Baltimore, MD, USA \\ ${ }^{4}$ Cahill Center for Astronomy and Astrophysics, California Institute of Technology 1200 E. California Boulevard, Pasadena, CA 91125, USA \\ ${ }^{5}$ Department of Physics and Astronomy, University of New Mexico, MSC07 4220, Albuquerque, NM 87131-0001, USA \\ ${ }^{6}$ U.S. Naval Observatory, Flagstaff Station, 10391 W. Naval Observatory Road, Flagstaff, AZ 86001, USA \\ Received 2013 September 9; accepted 2013 November 17; published 2013 December 19
}

\begin{abstract}
$4 \mathrm{C}+37.11$, at $z=0.055$, shows two compact radio nuclei, imaged by very long baseline interferometry at 7 mas separation, making it the closest known resolved supermassive black hole binary (SMBHB). An important question is whether this unique object is young and was caught on the way to a gravitational in-spiral and merger, or has "stalled" at $7 \mathrm{pc}$. We describe new radio/optical/X-ray observations of the massive host and its surrounding X-ray halo. These data reveal X-ray/optical channels following the radio outflow and large scale edges in the X-ray halo. These structures are promising targets for further study which should elucidate their relationship to the unique SMBHB core.
\end{abstract}

Key words: galaxies: active - galaxies: halos - X-rays: galaxies

Online-only material: color figures

\section{INTRODUCTION}

Supermassive black hole binaries (SMBHBs) are expected to form during hierarchical assembly of large galaxies (Begelman et al. 1980). Yet, while the evidence for major mergers is now strong (e.g., Bell et al. 2006), the evidence for SMBHBs is much more limited. Some double X-ray nuclei have been identified at kpc-scale separations (e.g., Owen et al. 1985; Komossa et al. 2003), but at these scales the holes may not be bound by mutual gravitational interaction. Indirect evidence for binarity at small separation has also been inferred from QSO emission line structure (e.g., Boroson \& Lauer 2009), although other interpretations remain viable in many cases (Eracleous et al. 2012, and references therein). To date, the only spatially resolved SMBHB with a parsec scale separation is the nucleus of the compact symmetric object (CSO) radio galaxy 4C+37.11. This galaxy contains a pair of unresolved, variable, non-thermal radio sources with a projected separation of $7 \mathrm{pc}$ (Maness et al. 2004; Rodriguez et al. 2006).

Attempts to find other such systems have not yet yielded fruit. For example, Burke-Spolaor (2011), in a review of multifrequency very long baseline interferometry (VLBI) maps of over 3000 active galactic nuclei (AGNs), confirmed that only $4 \mathrm{C}+37.11$ shows strong evidence for a resolved double core. Evidently, the rarity of such objects means that transition is relatively rapid from kpc-scale binaries to sub-pc scale where the merger lifetime to gravitational radiation losses is guaranteed to be short. Given the inadequacy of simple dynamical friction (Begelman et al. 1980), this raises some interesting theoretical questions. It also has important observational implications for the energy injection into the host nucleus (Milosavljevic \& Merritt 2003; Springel et al. 2005) and for the background

\footnotetext{
7 Visiting Astronomer, Kitt Peak National Observatory, which is operated by the Association of Universities for Research in Astronomy (AURA) under cooperative agreement with the National Science Foundation. The WIYN Observatory is a joint facility of the University of Wisconsin-Madison, Indiana University, Yale University, and the National Optical Astronomy Observatory.
}

of ultra-low frequency gravitational radiation that may be detectable with space-based (Hughes 2003) or pulsar timing (Detweiler 1979) searches. More specifically, it leads us to ask why $4 \mathrm{C}+37.11$ is uniquely seen at small separation. If not simple observational selection or fortuitous timing, perhaps some peculiarity of the system has delayed merger. Given the strong expected feedback between the AGN nucleus and the host environment (Cattaneo et al. 2009), we can hope that studying the $4 \mathrm{C}+37.11$ environment may yield useful clues.

In our initial study of the system we were only able to supplement the centimeter-wavelength VLBI maps with limited multi-wavelength data. For the optical we had only sky survey imaging, a shallow $R$-band frame in Stickel et al. (1993), and spatially unresolved Hobby-Eberly Telescope (HET) spectroscopy. However, $4 \mathrm{C}+37.11$ was detected as a resolved X-ray source by ROSAT (Voges et al. 1999), suggesting a unique (for a CSO) host environment. The situation called for a deeper multi-wavelength study. Here we report on observations of the host spanning the radio to X-ray regimes. These observations (Section 2) were obtained with the Karl G. Jansky Very Large Array (VLA), the WIYN $3.5 \mathrm{~m}$ telescope (optical imaging), the W. M. Keck I $10 \mathrm{~m}$ telescope (optical spectra) and the Chandra $X$-ray Observatory (X-ray imaging spectroscopy). Our analysis has indeed uncovered an unusual host morphology and evidence for AGN-host feedback. However further work will be needed to obtain definitive constraints on the merger age and current evolution of the SMBHB.

\section{OBSERVATIONS}

\subsection{VLA Radio Imaging}

The mas- to arcsec-scale radio structure of $4 \mathrm{C}+37.11$ has been described by Maness et al. (2004) and Rodriguez et al. (2006, 2009). The two compact cores $\mathrm{C} 1$ and $\mathrm{C} 2$ suggested as the black hole nuclei lie at position angle $118^{\circ}$ (measured north through east). The fainter, western nucleus $\mathrm{C} 2$ is the origin of 


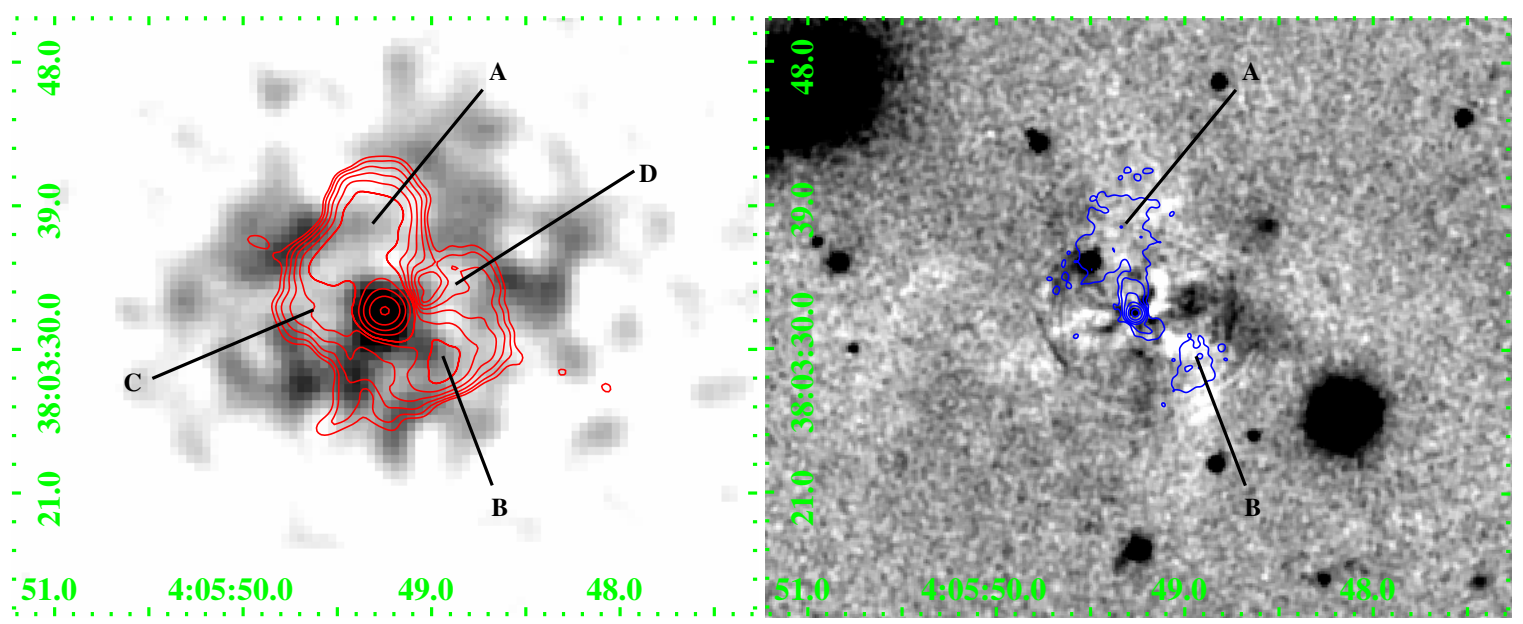

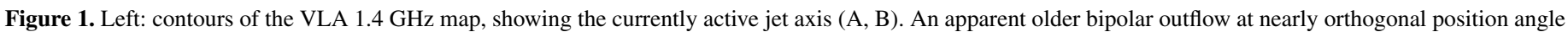

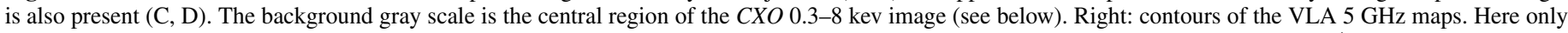

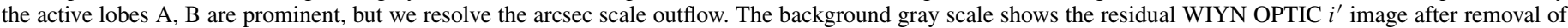
the host elliptical isophotes, shown with a linear intensity scale (see below).

(A color version of this figure is available in the online journal.)

the CSO outflow, visible as two relativistically expanding jets. The northern jet at P.A. $\approx 25^{\circ}$ seems to curve toward P.A. $\sim 0^{\circ}$ at the arcsecond scale. The southern jet lies at angle $205^{\circ}$ out to $\sim 50$ mas, then bends toward $225^{\circ}$ at $\sim 5 \operatorname{arcsec}$. In the $4.9 \mathrm{GHz}$ map of Maness et al. (2004) there also appears to be a weaker axis of emission with extensions at P.A. $\approx 80^{\circ}$ and $240^{\circ}$.

To trace these structures to arcsecond scales, we obtained VLA observations in the A configuration on 2012 November 11 (Proposal Code VLA/12B-058). Integrations were obtained in bands centered at $1.3 \mathrm{GHz}$ (effective bandwidth $384 \mathrm{MHz}, \mathrm{rms}$ noise $50 \mu \mathrm{Jy}_{\text {beam }}{ }^{-1}$, resolution $1.39 \times 1^{\prime \prime} .25$ at P.A. $=-81^{\circ}$ ) and $4.7 \mathrm{GHz}$ (effective bandwidth $256 \mathrm{MHz}$, rms noise $70 \mu \mathrm{Jy}$ beam $^{-1}$, resolution 0.5$)$.

All processing was performed in AIPS (Greisen 2003). Following editing using RFLAG and after discarding some channels with low signal levels, we fringe-fit the strong calibrator 3C286 to remove any residual delay errors. Next the data were amplitude calibrated using the AIPS tasks SETJY, CALIB, and GETJY. Bandpass calibration was determined from 3C286 while phase and polarization leakage calibration were obtained from observations of the nearby calibrator J0414+3418. Following calibration, imaging was carried out in AIPS taking advantage of new multi-frequency synthesis routines.

We show contours from the maps obtained in Figure 1, overlaid on the host emission at higher energy. The $4.7 \mathrm{GHz}$ contours show the main CSO outflow from nucleus C2 (A, B) which appears to follow optical "channels" in the host galaxy. At lower frequency the outflows in these channels are unresolved, but the lobes (A, B) appear to extend to $\sim 15^{\prime \prime}$ and occupy cavities in the host X-ray map. A second outflow axis $(C, D)$ at roughly orthogonal position angle also appears to correlate with $\mathrm{X}$-ray cavities. This may be an extension of the VLBA-measured secondary axis of Maness et al. (2004).

From a spectral index map made between 1.3 and $4.7 \mathrm{GHz}$ (not shown), we see that the core emission is very flat $(\alpha \approx 0)$, while the lobes are much steeper $(\alpha \approx-1.3$ to -1.7$)$, as seen in other radio galaxies in dense environments. The $\mathrm{C}$, D structures are particularly steep, being visible only at low frequency. The core of $4 \mathrm{C}+37.11$ itself appears to be unpolarized with a limit derived from Stokes $Q$ and $U$ images of $<0.2 \%$. For the weaker lobes we constrain the polarization to be $<1 \%$. It is likely that this represents de-polarization by the extensive cluster gas. We did observe significant polarization in three background sources, but the nearest of these was $\sim 8^{\prime}$ away, so this reveals little about the cluster gas or magnetic field.

\subsection{WIYN Optical Imaging}

In Figure 2 (left panel) we show a $2220 \mathrm{~s}$ stack of $i^{\prime}$ exposures taken on 2008 December 12 using the OPTIC orthogonal frametransfer camera (Tonry et al. 2002, 2004) on the Kitt Peak National Observatory, $3.5 \mathrm{~mm}$ Wisconsin, Yale, Indiana, and NOAO (hence WIYN) telescope. The frame-transfer allows rapid electronic guiding. The data were reduced with standard IRAF routines (Tody 1986; Valdes 1986), except that the flat field was dithered following the fast guiding corrections. The dithering code, conflat2, was provided by J. Tonry (2011, private communication). The combined image has a stellar FWHM resolution of $0{ }^{\prime} 39$. The host galaxy for $4 \mathrm{C}+37.11$, centered in the frame, has an integrated $i^{\prime}$ magnitude of 15.1. The next brightest galaxy lies $25^{\prime \prime} .3$ (projected distance $26.7 \mathrm{kpc}$ ) from the host core and is $2.5 \mathrm{mag}$ fainter at $i^{\prime}=17.6$. The next closest bright galaxies are at projected separations (and magnitudes) of $28^{\prime \prime} .2\left(i^{\prime}=19.7\right), 31^{\prime \prime} .5\left(i^{\prime}=18.6\right)$, and $31^{\prime \prime} .7\left(i^{\prime}=18.5\right)$. While we do not have spectroscopic confirmation that these galaxies are associated, the optical and X-ray morphology do show some evidence for interaction. For comparison, the middle panel shows diffuse X-ray emission at the same scale while the right panel of Figure 2 shows the large scale X-ray halo.

The Schlegel et al. (1998) maps give a large extinction $A_{V}=3.52, A_{i^{\prime}}=2.25$ in this direction. This gives a corrected host magnitude $i^{\prime}=12.8$. The Two Micron All Sky Survey (2MASS) data give extinction-corrected total host magnitudes of $J=11.34$ and $K_{s}=9.94$. Thus this is a very luminous galaxy, some $\sim 25 \times$ more luminous than $L^{*}$ : the extinctioncorrected absolute magnitudes are $M_{i^{\prime}}=-24.1\left(M_{i^{\prime}}^{*}-3.7\right)$, $M_{J}=-25.6\left(M_{J}^{*}-3.3\right)$, and $M_{K_{s}}=-27.0\left(M_{K_{s}}^{*}-3.5\right)$.

We have performed an isophotal analysis of the host using the IRAF task "ellipse." The surface brightness (Figure 3) shows a reasonable fit to a de Vaucoleurs profile; however, there is a modest step in surface brightness at a radius of $\sim 10 \mathrm{kpc}$. Our good resolution allows us to extend the analysis well within $1 \mathrm{kpc}$, where we see an appreciable flattening of the galaxy 


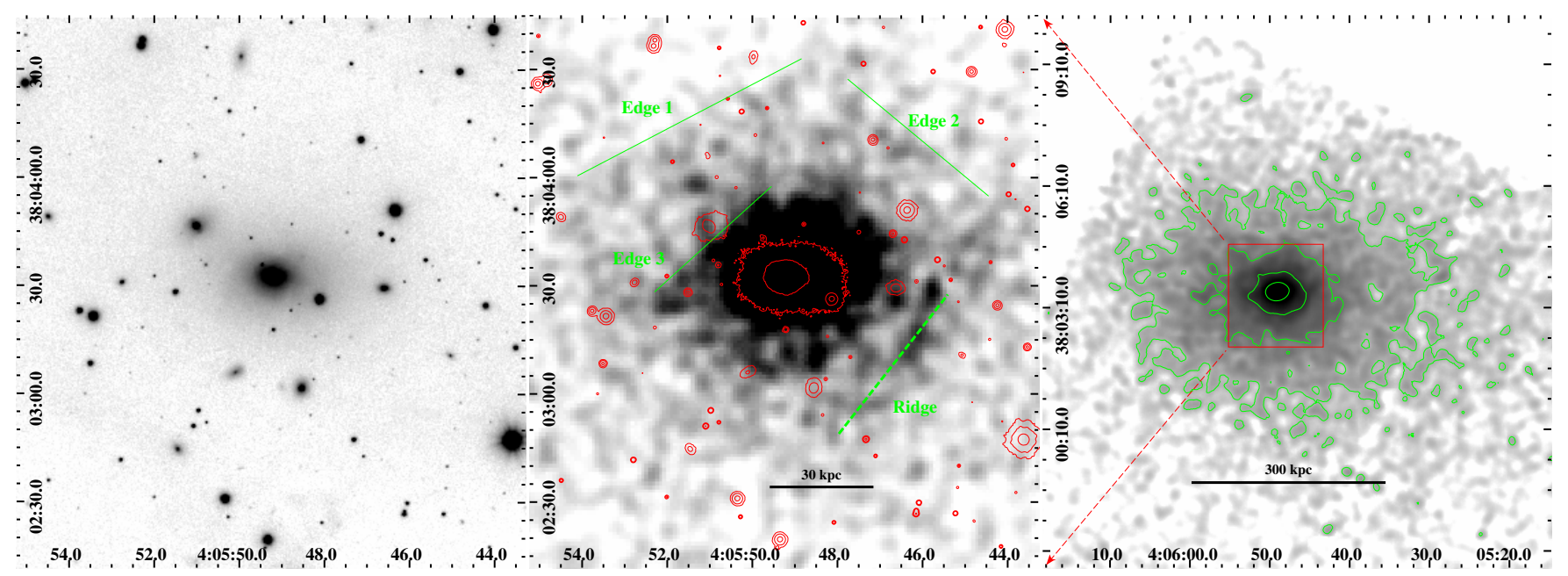

Figure 2. Left: WIYN/OPTIC $i^{\prime}$ image of $4 \mathrm{C}+37.11$. Middle: $0.3-8 \mathrm{keV}$ ACIS-I image, smoothed with a 4 " Gaussian. The contours mark the optical objects from the left frame, while lines indicate some diffuse X-ray features. Right: large scale 0.3-8 keV emission, exposure corrected and smoothed with a $12^{\prime \prime}$ Gaussian kernel, $\log$ stretch. The position of the left optical/X-ray frame is shown by the red box. North is up, east is to the left.

(A color version of this figure is available in the online journal.)

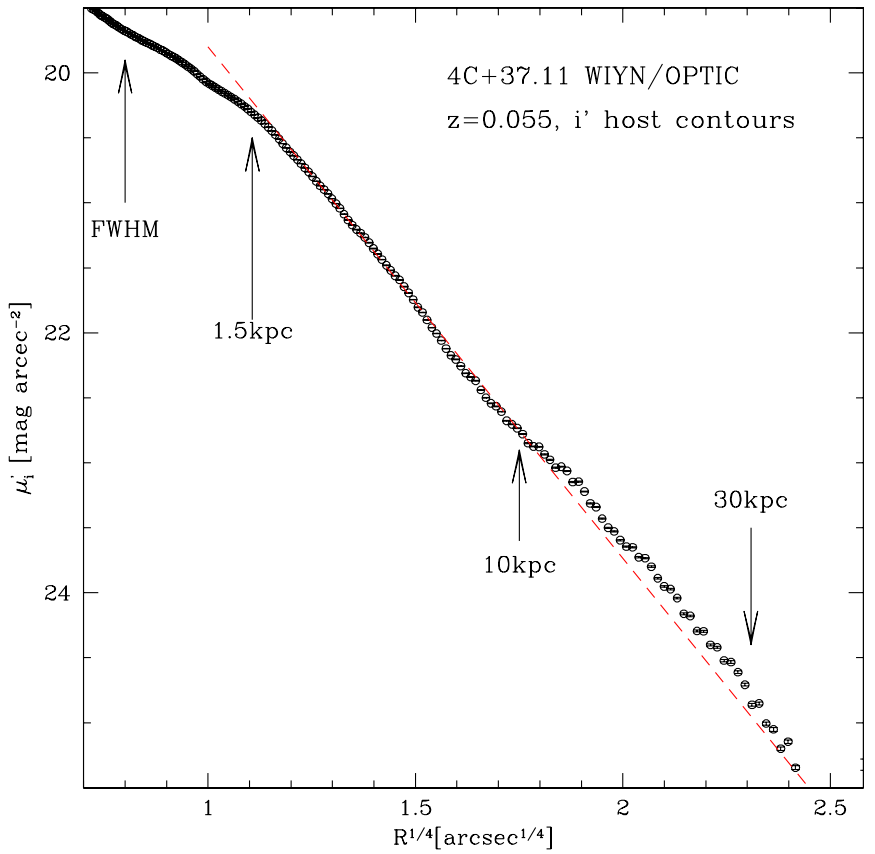

Figure 3. Host surface photometry averaged over elliptical isophotes. A reasonably good approximation to a de Vaucoleurs profile obtains a large radius, with no evidence for a $\mathrm{cD}$ tail. The step at $\sim 10 \mathrm{kpc}$ shows the extent of the cavities associated with the radio lobes. The inner kpc region shows strong flattening, partly associated with the channels evacuated by AGN outflow.

(A color version of this figure is available in the online journal.)

core. Since there is no evidence for a large excess to the $R^{1 / 4}$ law at large radii, we infer that this is not a $\mathrm{cD}$ galaxy.

We have subtracted a smooth galaxy model generated from these elliptical isophotes. Some elliptical residuals remain at $\sim 5^{\prime \prime}$ radii, but the subtraction shows considerable real structure (Figure 1). Most prominent are low surface brightness (here light) channels extending north and south to $\sim 10^{\prime \prime}$. These appear connected to and extend the VLA-scale radio lobes. A third channel extends to the east. All are tortuous. On smaller $<2^{\prime \prime}$ scales there appear to be two white, but edge-brightened channels oriented approximately east-west. The nature of these structures is unclear. They are approximately orthogonal to the VLBI-scale jet axis and so may represent equatorial obscuration. Alternatively, these may be channels evacuated by the secondary flow axis visible in the VLA maps. Additional high resolution images, with color information on sub-arcsec scales could help elucidate the nature of these channels, distinguishing emission line structure and dust obscuration from host cavities.

\subsection{Keck LRIS Spectroscopy}

Two exposures totaling $1200 \mathrm{~s}$ were obtained with the Keck I/LRIS on 2010 December 17. The red channel used the 400 line $/ 8500 \AA$ blaze grating for a dispersion of $1.18 \AA \mathrm{pixel}^{-1}$. We used a 1 arcsec slit positioned approximately along the VLBI jet axis (at P.A. $=20^{\circ}$, see right panel of Figure 1), giving an effective spectral resolution of $5.4 \AA$ and a spatial resolution of $0 ! 27 /$ (binned) pixel. With the $5600 \AA$ dichroic the red channel data covered 5500-10300 $\AA$. The $\mathrm{H} \alpha$ /forbidden lines near rest $6500 \AA$ i were well measured (Figure 4), and we detected the nebular [S III] 9069/9532 ̊ lines at 9570/10054 ̊. With the large extinction in this direction the spectra were highly reddened and little light was collected from the blue channel.

The lines are resolved, with an S-shaped velocity profile along the slit. To the north, the radial velocities are $\sim 300 \mathrm{~km} \mathrm{~s}^{-1}$ smaller than to the south of the nucleus. The nature of these line shifts are not clear; to the north the velocities seem to return to near the systemic velocity by about $2^{\prime \prime}$; to the south the offset persists. Since our position angle is close to the host minor axis, the velocities probably do not represent global rotation. They may instead represent velocity perturbations in the host interstellar medium associated with the outflow along the VLBIscale jet axis. This is at least consistent with the VLBI jet flux ratio near the core (Rodriguez et al. 2009), where the northern jet is brighter near its base and thus may be emerging from the plane of the sky. In Rodriguez et al. (2006) the line splitting of $\sim 300 \mathrm{~km} \mathrm{~s}^{-1}$ was noticed in the $2^{\prime \prime}$ HET spectra, and it was suggested that these might represent the radial velocities of the two BHB cores. At the projected separation of $7.3 \mathrm{pc}$ this indicated an enclosed mass of $>1.5 \times 10^{8} M_{\odot}$. It now seems likely that this integrated profile shape was caused by the larger scale outflows resolved here. However, Rodriguez et al. (2009) found $\mathrm{H}_{\mathrm{I}}$ absorption velocities differing by $1000 \mathrm{~km} \mathrm{~s}^{-1}$ over 

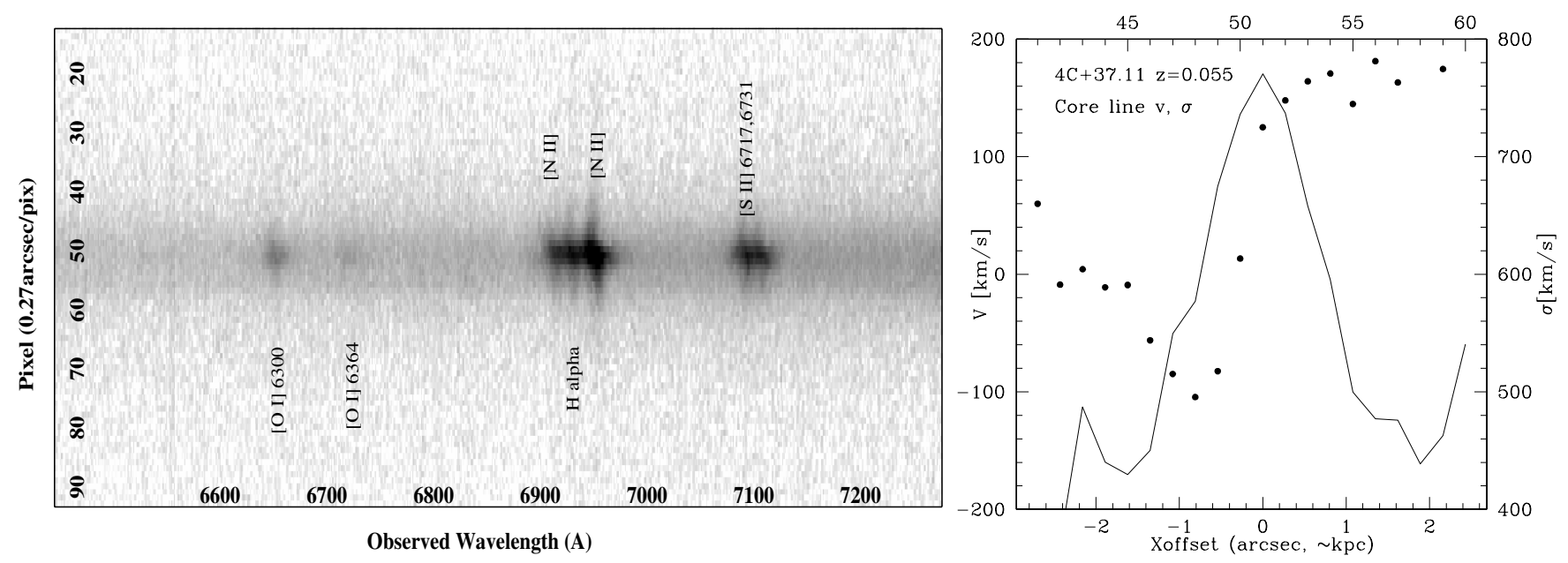

Figure 4. Keck LRIS long slit spectra of the central region of $4 \mathrm{C}+37.11$. The core shows highly reddened forbidden line emission, which displays velocity shifts within an arcsecond of the nucleus. The right panel shows the line shifts (dots, left scale) and broadening (line, right scale) of the $\mathrm{H} \alpha / \mathrm{N}[\mathrm{II}] \mathrm{complex}$.

$\sim 7$ mas in front of the southern jet, and used this to derive a lower mass limit of $7 \times 10^{8} M_{\odot}$.

The core itself is not spatially resolved with the $\sim 1^{\prime \prime}$ seeing during our spectroscopic exposures, but at the core the central velocity dispersion peaks at $\sim 750 \mathrm{~km} \mathrm{~s}^{-1}$, after correcting for the instrumental resolution. This suggests a mass of $\sim 7 \times 10^{10} M_{\odot}$ in the central $0.5 \mathrm{kpc}$, which easily accommodates the VLBI-scale mass estimates above.

\subsection{CXO ACIS Imaging}

As discussed in Rodriguez et al. (2006), 4C+37.11 was detected in the RASS and had two HRI observations, showing an extended X-ray halo. More recently a short $3 \mathrm{ks}$ SWIFT $\mathrm{X}$-ray telescope exposure confirmed diffuse X-rays extending several arcmin from the host galaxy and allowed an estimate of $k T \approx 4 \mathrm{keV}$ for the $\mathrm{X}$-ray halo temperature. To improve the spectral measurements and to get a first look at the arcsec-scale $\mathrm{X}$-ray structure of $4 \mathrm{C}+37.11$ we obtained an exploratory $C X O$ ACIS-I image on 2011 April 4 (ObsID 12704; PI: Murray). Using a Science Instrument Module (SIM) offset the focal point and target were shifted onto the I 3 chip, $>3^{\prime}$ from the array gaps so that the bulk of the diffuse halo would be covered on a single chip. The timed exposures used the standard $3.1 \mathrm{~s}$ frame time and were read out in VFAINT mode; we were able to use the full $10 \mathrm{ks}$ exposure as there were no strong flaring events during this observation. This exposure netted $\sim 16,000$ counts $(0.3-10 \mathrm{keV})$ from the X-ray halo and $\sim 30$ counts from the AGN itself.

The data were subject to standard CIAO 4.3 processing. In Figure 2, right, we show the large-scale distribution of the $\mathrm{X}$ ray halo with a smoothed, exposure corrected $0.3-8 \mathrm{keV}$ image. The X-ray halo is elliptical with an axis ratio $a / b \sim 1.33$, and P.A. similar to that of the central galaxy. It can be detected to a semimajor axis of at least $10^{\prime}$ (about $1100 \mathrm{kpc}$, or $0.6 \times r_{500}$, where the density drops to $500 \rho_{\text {crit }}$ as estimated in Section 3), although we lack the signal-to-noise ratio $(\mathrm{S} / \mathrm{N})$ for a study beyond $\sim 7^{\prime}$. The inner X-ray isophotes are boxy, suggesting edges in the surface brightness. In the middle panel of Figure 2, we show the central 2.5 arcmin, stretched to emphasize some of the diffuse features. "Edge 1" passes 50 " NE of the central galaxy and has a count rate surface brightness contrast of $\sim 2.5 \times$. "Edge 2," $55^{\prime \prime}$ to the NW is somewhat less well defined, but still seems to represent a $\sim 2 \times$ step. "Edge 3," 20 " from the host core is centered just inside the nearest bright companion galaxy and with a $\sim 3 \times$ intensity step may represent a front associated with companion interaction. On the opposite $\mathrm{SW}$ side at $\sim 35^{\prime \prime}$ lies an apparent ridge of X-ray emission. Similar, albeit less well defined, structures are visible at both larger (Figure 2, right) and smaller (Figure 1, left) scales. Unfortunately we lack sufficient counts to measure these structures with high accuracy or to determine if these are shock fronts or cold fronts. However, clearly the halo of $4 \mathrm{C}+37.11$ shows extensive disturbance and abundant evidence of past interactions. A deeper X-ray image is needed for an adequate spatial/spectral study of these structures.

Turning to smaller scales, we can see a good correspondence between the radio outflow and the X-ray surface brightness (Figure 1). In particular the northern radio lobe occupies a welldefined cavity in the X-rays. There also seems to be decreased X-ray surface brightness along the east-west cavities of the secondary outflow axis. However the southern radio lobe, which shows a marked cavity in the optical, does not appear well defined in the X-ray.

\subsection{CXO X-Ray Spectroscopy}

For the AGN itself, we detect only $30 \pm 6$ counts, embedded in a bright structured background. These counts are hard with $1 / 3$ of the background-subtracted photons at $E>$ $4 \mathrm{keV}$. We cannot distinguish heavy core absorption from hard spectrum nuclear emission but if we assume $N_{\mathrm{H}}=8 \times$ $10^{21} \mathrm{~cm}^{-2}$, as determined from the halo emission (below), and a $\Gamma=1$ power law index, a Sherpa fit gives a $2-10 \mathrm{keV}$ flux $6.3 \pm 2.4 \times 10^{-14} \mathrm{erg} \mathrm{cm}^{-2} \mathrm{~s}^{-1}$. Thus we can only conclude that the emission is consistent with that of a typical Seyfert core. In contrast for the diffuse halo we have sufficient counts to estimate the temperature in a handful of regions and to get the radial gradient in the emissivity.

For an initial study of the halo, we defined a series of elliptical shells centered on the AGN core, with semimajor axes $\theta_{\text {maj }}=\left(20 \times 2^{N}\right)^{\prime \prime}$ and semiminor axes $0.75 \times \theta_{\text {maj }}$, with north taking values $0,1 \ldots 4$. We fit various extraction apertures using Sherpa. For all fits we used a background region $\sim 14^{\prime}$ from the $\mathrm{X}$-ray core on the I0 chip. This is along the halo minor axis at a projected distance corresponding to $\sim 1.0-1.3 r_{500}$; while this represents the maximum distance accessible with the active chips there may be some suppression of the lowest surface brightness flux estimates. Spectra were grouped to maintain 


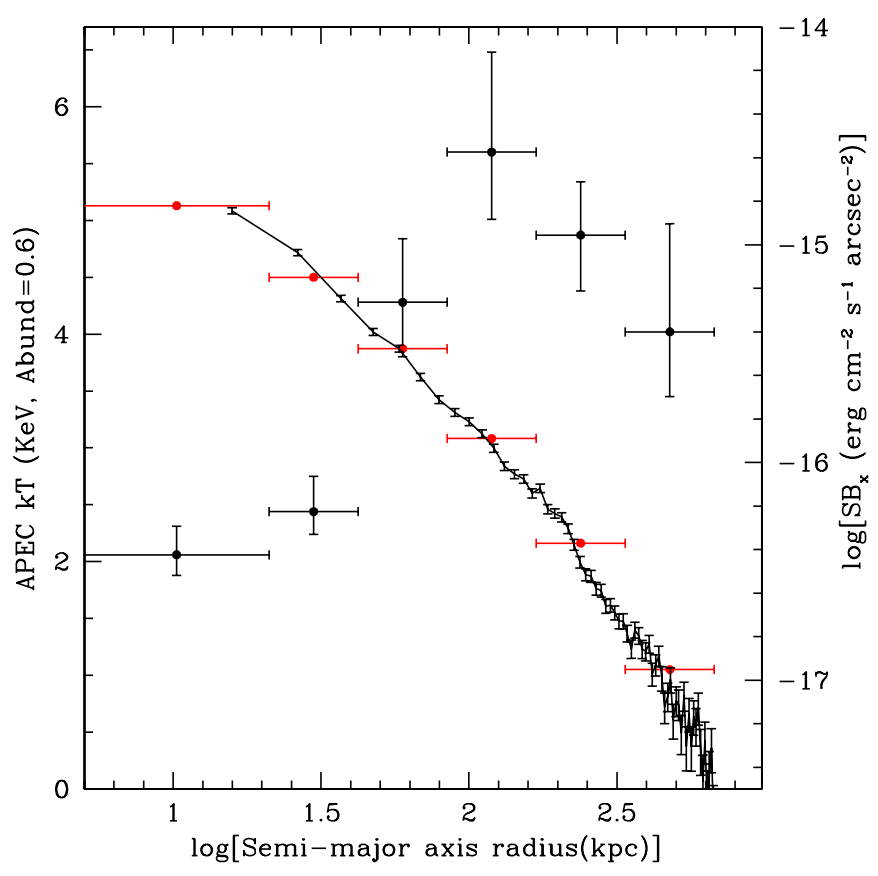

Figure 5. Line shows the elliptical (aspect ratio 4:3) surface brightness profiles of the halo X-rays. We also show the best-fit mean surface brightness (points with horizontal flags, right scale) and temperature (horizontal and vertical flags, left scale) in our six zones (Table 1). Note the strong cooling in the halo core.

(A color version of this figure is available in the online journal.)

$\mathrm{S} / \mathrm{N}=3 /$ bin and the source was assigned a model redshift $z=0.055$. All quoted errors are $1 \sigma$ single parameter values.

We first fit the sum of regions 2-4 (excluding the cooled core) to an absorbed APEC model, obtaining an overall temperature $k T_{\mathrm{sp}}=4.8 \pm 0.2 \mathrm{keV}$ and an abundance (relative to solar) of $\mathrm{Ab}=0.63 \pm 0.10$. The absorption was found to be $N_{\mathrm{H}}=8.2 \pm 0.4 \times 10^{21} \mathrm{~cm}^{-2}$. This gives an equivalent $A_{V}=N_{\mathrm{H}} / 2.2 \times 10^{21} \mathrm{~cm}^{-2}=3.7$, in excellent agreement with the full Galactic extinction estimated from the Schlegel et al. (1998) maps, suggesting that the intrinsic absorption is small. The fit source flux out to $\theta_{\text {maj }}=320^{\prime \prime}\left(\approx 340 \mathrm{kpc} \approx r_{500} / 3\right)$ is $f_{x}(0.5-8 \mathrm{keV})=1.83 \pm 0.07 \times 10^{-11} \mathrm{erg} \mathrm{cm}^{-2} \mathrm{~s}^{-1}$. The fit is quite acceptable with $\chi_{\text {Geh }}^{2} /$ dof $=222 / 341$.

We next fixed the extinction and abundance at the global values and fit the temperature and flux in the elliptical zones using the Sherpa deproject script, starting from the outermost shell. This script accounts for the emission from larger shells to reconstruct a true three-dimensional temperature and flux estimate. We see that the halo temperature peaks at about $2^{\prime}(\sim 100 \mathrm{kpc}$; Figure 5). We also show the surface brightness determined by the de-projection fit to the elliptical shells and compare with a simple profile of the count rate $\operatorname{arcsec}^{-2}$, where the latter has been normalized by the effective efficiency $6.9 \mathrm{keV}$ count $^{-1}$. While the halo is detectable to well beyond the temperature peak, there are no dramatic steps in the azimuthalaveraged surface brightness profile.

Although we lack the statistics to do a full de-projection analysis, if we use simple annuli to fit the abundance we do detect a marginally significant gradient with $\mathrm{Ab}=1.0 \pm 0.3$ in the central cooling core, and a drop off to $\mathrm{Ab}=0.3 \pm 0.2$ in the outermost regions.

We have also attempted to fit the apparent edges shown in Figure 1, by estimating the temperature and flux in rectangular regions bracketing the steps, but again lack the counts for a definitive characterization. For Edge 1 the spectra fits show
Table 1

X-ray Spectral Fits to the Halo of $4 \mathrm{C}+37.11$

\begin{tabular}{lcccc}
\hline \hline$N$ & $\begin{array}{c}\theta_{\text {maj }} \\
\left({ }^{\prime \prime}\right.\end{array}$ & $\begin{array}{c}k T \\
(\mathrm{keV})\end{array}$ & $f_{0.5-8}{ }^{\mathrm{a}}$ & $\chi^{2 / \mathrm{dof}}$ \\
\hline 0 & 20 & $2.05_{-0.18}^{+0.25}$ & $1.51_{-0.09}^{+0.09}$ & $69 / 112$ \\
1 & 40 & $2.44_{-0.20}^{+0.31}$ & $2.06_{-0.14}^{+0.12}$ & $68 / 127$ \\
2 & 80 & $4.28_{-0.48}^{+0.56}$ & $3.89_{-0.19}^{+0.22}$ & $98 / 195$ \\
3 & 160 & $5.60_{-0.59}^{+0.88}$ & $6.04_{-0.25}^{+0.24}$ & $173 / 263$ \\
4 & 320 & $4.87_{-0.49}^{+0.47}$ & $7.90_{-0.26}^{+0.26}$ & $146 / 324$ \\
5 & 640 & $4.02_{-0.57}^{+0.95}$ & $4.30_{-0.28}^{+0.25}$ & $160 / 344$ \\
\hline
\end{tabular}

Note. ${ }^{a}$ Absorbed flux in $10^{-12} \mathrm{erg} \mathrm{cm}^{-2} \mathrm{~s}^{-1}$.

(moving outward) a decrease in surface brightness from $\mathrm{SB}_{X}=$ $3.9 \pm 0.3$ to $1.4 \pm 0.1$ (in units of $10^{-16} \mathrm{erg} \mathrm{cm}^{-2} \mathrm{~s}^{-1} \operatorname{arcsec}^{-2}$ ) or a $2.8 \pm 0.3 \times$ drop. The temperature step (from $5.8_{-1.2}^{+2.1} \mathrm{keV}$ to $6.4_{-1.3}^{+2.1}$ ) was not, however, significant. In the core for Edge 3 we find a surface brightness drop of $\mathrm{SB}_{X}=11.8 \pm 1.1 \longrightarrow 4.4 \pm 0.3$ $(2.7 \pm 0.3 \times)$ while the temperature increase $3.4_{-0.5}^{+0.6} \mathrm{keV}$ to $4.5_{-0.7}^{+1.0} \mathrm{keV}$ is only marginally significant.

\section{THE HOST ENVIRONMENT AND MASSES}

It is useful to constrain the mass of $4 \mathrm{C}+37.11$ 's host environment. The host galaxy itself has $M_{K}=-27.0$ $\left(L=1.3 \times 10^{12} h_{70}^{-2} L_{\odot}\right)$, or $M \approx 9.5 \times 10^{11} M_{\odot}$. This is a massive elliptical. The dynamical time for this mass and a radius of $\sim 20 \mathrm{kpc}$ is about $60 \mathrm{Myr}$. There has been ample time for the stellar distribution to be smoothed after a merger into the surface brightness profile seen in our WIYN photometry. The halo X-ray luminosity $1.2 \times 10^{44} \mathrm{erg} \mathrm{s}^{-1}$ is also large. Thus the host components, while luminous, have a $L_{X} / L_{\text {opt }} \approx 40$ ratio not atypical for the brightest cluster galaxy (BCG; Voevodkin et al 2010).

The X-rays give a more direct measurement of the largerscale potential. Vikhlinin et al. (2006) have provided convenient scaling relations for low- $z$ clusters:

$$
\begin{gathered}
r_{500}=0.792 h^{-1} \operatorname{Mpc}\left(T_{\mathrm{sp}} / 5 \mathrm{keV}\right)^{0.53} \text { and } \\
M_{500}=2.89 \times 10^{14} h^{-1} M_{\odot}\left(T_{\mathrm{sp}} / 5 \mathrm{keV}\right)^{1.58} .
\end{gathered}
$$

With our fit values and adopted cosmology these are $r_{500}=1.1$ Mpc and $M_{500}=4 \times 10^{14} M_{\odot}$. Alternatively we can extrapolate from the extended flux within $400 \mathrm{kpc}\left(\approx r_{2500}\right.$, see Ascasibar et al. 2006) to obtain an unabsorbed $f_{x}(0.5-2 \mathrm{keV})=2.1 \times$ $10^{-11} \mathrm{erg} \mathrm{cm}^{-2} \mathrm{~s}^{-1}$ and an X-ray luminosity $L_{x}(0.5-2 \mathrm{keV})=$ $1.5 \times 10^{44} \mathrm{erg} \mathrm{s}^{-1}$. For this $L_{x}$ the scaling relations in Vikhlinin et al. (2009) give $M_{500} \approx 1.4 \times 10^{14} M_{\odot}$.

Lin \& Mohr (2004) have noted a correlation between the BCG $K$-band luminosity and cluster mass $M_{200} \approx 1.36 M_{500}$,

$$
M_{200}=\left(L_{\mathrm{BCG}} / 2.2 \times 10^{11} h^{-2} L_{\odot}\right)^{3.0 \pm 0.6} 10^{14} M_{\odot} .
$$

For our $K_{s}$ luminosity above this gives an exceptionally large $M_{500}=1.9 \times 10^{15} M_{\odot}$. Since this is much larger than the direct halo mass estimates, it suggests that the central galaxy may be somewhat anomalous compared to the set studied in Lin \& Mohr (2004). Indeed the galaxy appears about $2.5 \times$ brighter than the trend line in that study, so further measurements are needed to see if the host elliptical has, e.g., excess luminosity from ongoing star formation. 
The next question is the nature of this halo. Is it a typical galaxy cluster or a "fossil" cluster? Voevodkin et al. (2010) give the following criteria for a fossil: for halos with $L_{x}>2.5 \times 10^{41} \mathrm{~h}^{-2} \mathrm{erg} \mathrm{s}^{-1}$, there should be a "magnitude gap" $\Delta m_{12}>1.7$ (in R) for all members within half the virial radius which we take as $0.7 r_{500} \approx 700 \mathrm{kpc} \approx 12^{\prime}$. Certainly the nearby galaxies in our $i^{\prime}$ image satisfy this criterion. Given the substantial extinction in this direction, a list of 2MASS extended sources with total $K$-band magnitude $K_{\text {tot }}<12.25$ within $0.7 r_{500}$ is a useful catalog check. There are two such sources, 2MASS $04050165+3801503$ with $K_{\text {tot }}=11.8$ at 9.5 and 2MASS $04051504+3806532$ with $K_{\text {tot }}=12.0$ at 7'.5 projected separation. Neither has a redshift so a spectroscopic exclusion of membership of (at least) these galaxies would be needed to confirm a fossil status for $4 \mathrm{C}+37.11$.

If it is a fossil, how long has it been since its last major merger? The presence of an X-ray structure shows that this system is not fully relaxed. However the central cavities, at least, can clearly be attributed to recent activity by the central AGN. Rodriguez et al. (2006) found velocities of $\sim 0.1 c$ for the 20 mas scale radio lobes, indicating an age of $\sim 10^{3}$ yr. If the bulk relativistic motion extends up to the full $\sim 5^{\prime \prime}$ scale of the VLA radio lobes, that would indicate a lifetime of $\sim 3 \times 10^{5} \mathrm{yr}$. However, given that the apparent jet bending indicates slowing by $>4 \times$ and that the $i^{\prime}$ host "channels" have a length approaching $30^{\prime \prime}$ it is likely that the source has remained active for $>3 \times 10^{6} \mathrm{yr}$.

On larger scales, the X-ray halo shows evidence for edge structures. Unfortunately with the present $\mathrm{S} / \mathrm{N}$ we cannot map their full extent, but such structures can represent the "sloshing" of cluster gas, which, through comparison with cluster hydrodynamics simulations, can be used to constrain the age of major merger events (Johnson et al. 2012, and references therein). Here the colder core gas of the main cluster, attracted by the sub-cluster's mass, takes a lagging orbit about the overall potential minimum, making a sub-sonic trail, ram pressure confined by the hotter halo gas from larger radii. A common sloshing pattern, for finite impact parameter mergers close to the plane of the sky, is a spiral of such "cold" front edges. Such structures first form $\sim 0.3 \mathrm{Gyr}$ after the first pass of the merging component and can persist for several Gyr. On intermediate $\tau_{\text {dyn }}$ timescales, if a merger event is supersonic there can also be shocks showing interaction between the main halo gas and that of a merging companion.

For $4 \mathrm{C}+37.11$, the general impression is a series of fronts (Edge 1, Edge 3, the Ridge) normal to an axis at P.A. $\approx 45^{\circ}$. This may represent a series of shells, rather than a large scale spiral. Such structure could be the residue of a zero angular momentum merger close to the plane of the sky or of a more typical merger spiral viewed nearly edge-on with the angular momentum axis near the plane of the sky. In any event our limited X-ray spectroscopic data do support the cold-front interpretation with higher temperatures following lower surface brightness. However, a deeper exposure is needed to see if this trend is a significant discontinuity beyond the general cooling gradient. Also a more sensitive mapping of the structure at other position angles (e.g., "Edge 2") is needed to determine the overall morphology and allow a comparison with simulations.

We should finally check what sort of black hole mass we expect for this giant elliptical host. Gueltekin et al. (2009) have provided updates to the $M_{\bullet}-\sigma$ and $M_{\bullet}-L$ relations:

$$
\begin{aligned}
& \log \left(M_{\bullet} / M_{\odot}\right)=8.23 \pm 0.08+(3.96 \pm 0.42) \log \left(\sigma / 200 \mathrm{~km} \mathrm{~s}^{-1}\right), \\
& \log \left(M_{\bullet} / M_{\odot}\right)=8.95 \pm 0.11+(1.11 \pm 0.18) \log \left(L_{V} / 10^{11} L_{\odot, v}\right) .
\end{aligned}
$$

For giant ellipticals at $z \approx 0.05$ we expect colors $V-K_{s}=3.10$ and $V-i=0.64$. Together with our absolute magnitude estimates, these give $V=-23.9$ and $V=-23.6$. Thus our host luminosities suggest a (total) hole mass of $\log M_{\bullet}=9.4-9.5$. This may be easily accommodated by the central velocities measured by our long slit spectroscopy.

\section{CONCLUSIONS}

$4 \mathrm{C}+37.11$ remains a unique source and a possible Rosetta stone for the understanding of major mergers and the eventual fate of the component's supermassive black holes (SMBHs). We have found here that the host of this SMBH binary is a remarkably luminous elliptical galaxy and that in turn this is embedded in a cluster-scale X-ray halo. A dearth of other bright galaxies suggests that this may be a "fossil" cluster. However, there are central structures in the X-ray surface brightness that imply that this system is not fully relaxed. In addition we have discovered remarkable "channels" in the host galaxy optical surface brightness that correlate well with the observed large scale radio outflow of the central engine. Clearly this is a case where "feedback" is active. Interestingly, these channels are quite twisted, covering a large range of position angles about the central engine. It is possible that this indicates a secondary outflow axis from the SMBHB or a precession or spin-flip variation caused by SMBH interaction. In any event it indicates relatively isotropic and, hence effective, mechanical heating of the central X-ray halo.

In addition we detect X-ray structures on larger $(\sim 10 \mathrm{kpc})$ scales. At present it remains unclear if these represent "sloshing" cold fronts or more recent shocks, but these likely preserve evidence of a past major merger event.

The next steps are to use high resolution color information to see whether the host and its central channel structures show evidence of young stellar populations or nebular emission. We can also improve our understanding of the sub-arcsecond scale structure with additional, spatially resolved spectroscopy. Finally, on larger scales, we could certainly profit from a deeper $\mathrm{X}$-ray image. While the present snapshot suffices to show that there is a rich X-ray structure and that it correlates with known radio and optical morphology, we lack the counts to map this in detail and to make a spectroscopic identification of the state of the gas. Given the unusual nature of this luminous halo and the possibility that it will shed light on the unmerged state of the SMBHB at the core, this is a compelling quest, and new observations to enable a detailed morphology/spectral study are underway.

This work was supported in part by NASA grants NNX10AD11G and NNX10AP65G (R.W.R.) and Chandra Award number GO2-13149X (G.B.T.) issued by the Chandra $\mathrm{X}$-ray Observatory Center, which is operated by the Smithsonian Astrophysical Observatory for and on behalf of NASA under contract NAS8-03060. We thank Adam van Etten for help with the CIAO reductions. R.T.Z. thanks M. Stickel for providing his imaging data of $4 \mathrm{C}+37.11$, S. Howell for assistance during the WIYN runs and K. Herrmann for helpful discussions on narrow-band imaging.

Some of the data presented herein were obtained at the W. M. Keck Observatory, which is operated as a scientific partnership among the California Institute of Technology, the University of California, and the National Aeronautics and Space Administration. The Observatory was made possible by the generous financial support of the W. M. Keck Foundation. 
The National Radio Astronomy Observatory is a facility of the National Science Foundation operated under cooperative agreement by Associated Universities, Inc.

Facilities: VLA, CXO (ACIS), WIYN (OPTIC), Keck:I (LRIS)

\section{REFERENCES}

Ascasibar, Y., Sevilla, R., Yepes, G., Müller, V., \& Gottlöber, S. 2006, MNRAS, 371,193

Begelman, M. C., Blandford, R. D., \& Rees, M. J. 1980, Natur, 287, 307

Bell, E. F., Phleps, S., Somerville, R. S., et al. 2006, ApJ, 652, 270

Boroson, T. A., \& Lauer, T. R. 2009, Natur, 458, 53

Burke-Spolaor, S. 2011, MNRAS, 410, 2113

Cattaneo, A., Faber, S. M., Binney, J., et al. 2009, Natur, 460, 213

Detweiler, S. 1979, ApJ, 234, 1100

Eracleous, M., Boroson, T. A., Halpern, J. P., \& Liu, J. 2012, ApJS, 201, 23

Greisen, E. W. 2003, in Information Handling in Astronomy-Historical Vistas, ed. A. Heck (Astrophysics and Space Science Library, Vol. 285; Dordrecht: Kluwer), 109

Gueltekin, K., Richstone, D. O., Gebhardt, K., et al. 2009, ApJ, 698, 198

Hughes, S. A. 2003, AnPhy, 303, 142

Johnson, R. E., ZuHone, J. A., Jones, C., Forman, W., \& Markevitch, M. 2012, ApJ, 751, 95
Komoosa, S., Burwitz, V., Hasinger, G., et al. 2003, ApJL, 582, L15

Lin, Y.-T., \& Mohr, J. J. 2004, ApJ, 617, 879

Maness, H. L., Taylor, G. B., Zavala, R. T., Peck, A. B., \& Pollack, L. K. 2004, ApJ, 602, 123

Milosavljevic, M., \& Merritt, D. 2003, ApJ, 560, 860

Owen, F. N., O’Dea, C. P., Inoue, M., \& Eilek, J. A. 1985, ApJL, 294, L85

Rodriguez, C., Taylor, G. B., Zavala, R. T., et al. 2006, ApJ, 646, 49

Rodriguez, C., Taylor, G. B., Zavala, R. T., Pihlström, Y. M., \& Peck, A. B. 2009, ApJ, 697, 37

Schlegel, D. J., Finkbinder, D. P., \& Davis, M. 1998, ApJ, 500, 525

Springel, V., DiMatteo, T., \& Hernquist, L. 2005, MNRAS, 361, 776

Stickel, M., Kuehr, H., \& Fried, J. W. 1993, A\&AS, 97, 483

Tody, D. 1986, Proc. SPIE, 627, 733

Tonry, J. L., Burke, B. E., Luppino, G., \& Kaiser, N. 2004, in Scientific Detectors for Astronomy, The Beginning of a New Era, ed. P. Amico, J. W. Beletic, \& J. E. Beletic (Astrophysics and Space Science Library, Vol. 300; New York, NY: Kluwer), 385

Tonry, J. L., Lupino, G. A., Kaiser, N., Burke, B., \& Jacoby, G. H. 2002, Proc. SPIE, 4836, 206

Valdes, F. 1986, Proc. SPIE, 627, 749

Vikhlinin, A., Burenin, R. A., Ebeling, H., et al. 2009, ApJ, 692, 1033

Vikhlinin, A., Kravtsov, A., Forman, W., et al. 2006, ApJ, 640, 691

Voevodkin, A., Borozdin, K., Heitmann, K., et al. 2010, ApJ, 708, 1376

Voges, W., Aschenbach, B., Boller, T., et al. 1999, A\&A, 349, 389 